\title{
Electric field induced slanting growth of silicon nanowires with enhanced hydrophobic property
}

\author{
Yanhong Zhao, ${ }^{1}$ Wenyao $\mathrm{Li}^{* 1}$, Zhe Wang ${ }^{1}$, Quanyi $\mathrm{Li}^{1}$, Guanjie $\mathrm{He}^{2 *}$ \\ ${ }^{1}$ School of material engineering, Shanghai University of Engineering Science, Shanghai 201620, \\ China. \\ ${ }^{2}$ Materials Chemistry Centre, Department of Chemistry, University College London, 20 Gordon \\ Street, London WC1H OAJ, U. K. \\ E-mail: 1iwenyao314@gmail.com; guanjie.he.14@ucl.ac.uk
}

\begin{abstract}
The etching process of Si nanowires under DC electric field was studied in this work. Interestingly, the growth direction of silicon nanowires became slanting when applied with DC electric intensity of $600 \mathrm{~V} / \mathrm{m}$, which greatly influenced the surface wettability ascribed to the variation of surface morphologies. The contact angle of slant Si nanowire was enhanced compared with vertical growth Si nanowire $\left(132.4^{\circ}\right.$ vs. $\left.86.8^{\circ}\right)$.
\end{abstract}

\section{Keywords}

Microstructure; Si Semiconductors; Electric field; Surfaces

\section{Introduction}

In natural world, numbers of fascinating materials and structured surfaces are built with excellent surface wettability [1]. Biomimetic investigations have demonstrated that some natural superhydrophobic phenomena, such as the de-wetting behavior of lotus in plantae [2] and superhydrophobicity of a water strider's leg in insect. [3] These superhydrophobic behaviors are related to their unique micro- and nanostructured surfaces, especially, the lotus leaf is one of the most well-known and 
studied object due to its superhydrophobicity and exhibits a high-water contact angle of around $161^{\circ}$. [4] To fabricate such unique surfaces, various technologies such as template synthesis, phase separation, electrochemical deposition, electro-hydrodynamics, sol-gel methods, chemical vapor deposition, self-assembly, etc. have been developed for the synthesis of advanced materials with controlled microstructures. [5-7] These researches have revealed that rough surfaces with micro or nanoscale structures play important roles in the realization of superhydrophobic surfaces.

According to some recent reports, the existence of homogeneous direct current (DC) electric field could influence the growth and micro-structure of crystals [8-10]. For instance, Lu et al.[11] investigated the effect of the DC electric field on the phase transitions of ferroelectric thin film. Zhao et al.[12] studied the solidification of the metal under DC electric field and concluded that the presence of DC electric field alters the electro-migration of metal ions as well as the size of metal crystal particles. These studies also confirmed that the existence of DC electric field could change the surface morphology of metal materials. Based on these researches, it is believed that the existence of the DC electric field could affects the formation of semiconductor materials at a certain extent. Unfortunately, the studies on the effects of the DC electric field on the structure and morphology variation have little been reported before.

In this paper, a very important semiconductor, i.e., silicon nanowires were studied with and without DC electric field under the etching process. Interestingly, the growth direction of silicon nanowires became slant when applied with electric intensity of $600 \mathrm{~V} / \mathrm{m}$, which greatly influenced the surface wettability ascribed to their surface morphology variation. The contact angle of slant Si nanowire was enhanced 
compared with vertical Si nanowire which is synthesized without electric field $\left(132.4^{\circ}\right.$ vs. $\left.86.8^{\circ}\right)$. Moreover, the mechanism of changing etching direction in the DC electric field is also illustrated.

\section{Experiment}

\subsection{Preparation of vertical Si nanowires}

The vertical Si nanowires were prepared according to the previous report [13]: the apparatus for synthesizing silicon nanowires is conventional Teflon-lined stainless steel autoclaves, which was filled with etching $\mathrm{HF}$ and $\mathrm{H}_{2} \mathrm{O}_{2}$ solution containing silver nitrate up to $80-85 \%$ of its total volume. Firstly, silicon wafer (Fig. S1) were cleaned with acetone and ethanol in order to remove organic grease. The degreased silicon wafers were then etched for 10 minutes in diluted aqueous HF solution. The treated wafers were immersed into the etching solution immediately and treated at $50^{\circ} \mathrm{C}$ for $60 \mathrm{~min}$. Here, the etching solutions contain $5.0 \mathrm{~mol} / \mathrm{L} \mathrm{HF}$ and $0.02 \mathrm{~mol} / \mathrm{L}$ silver nitrate. Finally, the obtained products were thoroughly cleaned with concentrated nitric acid solution for several hours to remove redundant $\mathrm{Ag}$ and then dried in vacuum for further characterization.

\subsection{Preparation of slanting Si nanowires}

The slanting Si nanowires were prepared using the similar method to the vertical ones except that the etching process was under the DC electric field. Fig. 1 shows the experiment device for slanting Si nanowires: A couple of conductive $\mathrm{Cu}$ foils were aligned fixed to the two sides of hydrothermal autoclaves so that the uniform electric field could pass through it, and the distance between the two $\mathrm{Cu}$ foils is about $5 \mathrm{~cm}$. The electric field was generated by DC stabilized voltage power supply and the electric intensity is $600 \mathrm{~V} / \mathrm{m}$. 


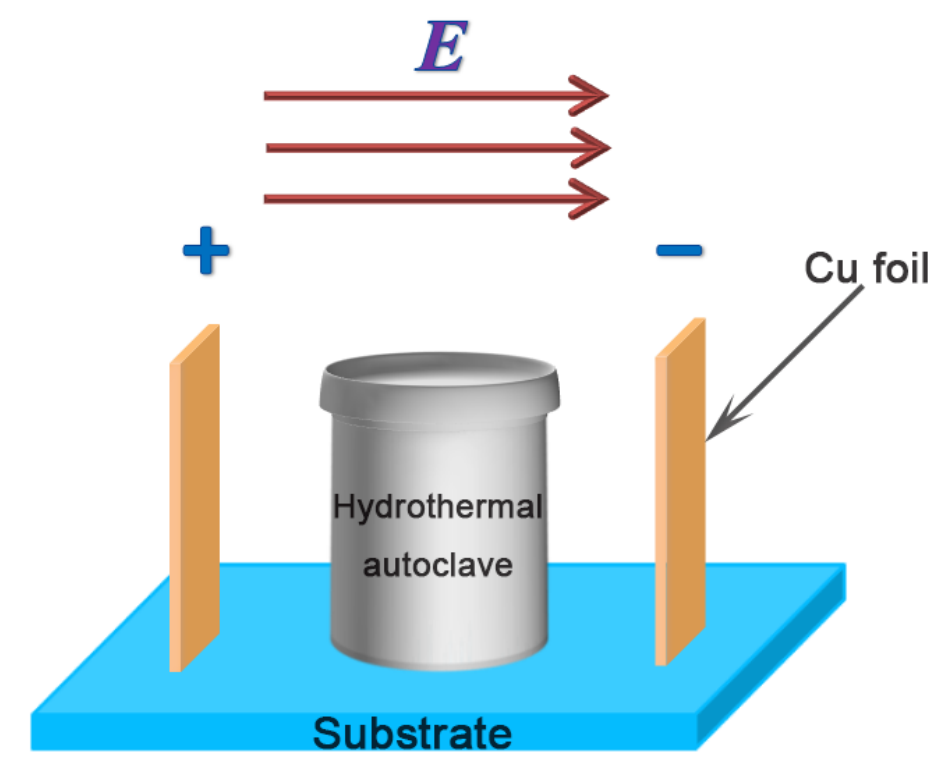

Fig. 1 Experimental device schematic for slanting Si nanowires

\subsection{Characterizations}

The SEM images of the silicon nanowires were obtained via a field-emission SEM apparatus (S-4800, Hitachi). The hydrophobic or hydrophilic performance of the silicon nanowires were measured by a JC2000D2A water contact angle tester (Zhongchen digital technic apparatus co., ltd, Shanghai, China).

\section{Results and discussion}

\subsection{Morphology analysis of Si nanowires}

Fig. 2a shows the cross-sectional SEM view of a p-type Si (100) wafer after 60 min of etching, which shows large-area aligned silicon nanowire arrays perpendicular to the Si surface. The length of silicon nanowire is $\sim 4 \mu \mathrm{m}$ with smooth surface. Contact angle (CA) measurements were carried out to evaluate the wettability of the Si nanowires. Inset of Fig. 2a shows that the CA of a water droplet on vertical Si nanowires surface is 86.8 degrees, which shows that the vertical Si nanowires are 
hydrophilic. Fig. $2 \mathrm{~b}$ shows the Si nanowires produced under the DC electric field. Interestingly, the grown Si nanowire arrays become slant with rough surface. Many pits were left on the surface of nanowires during the formation process (Fig. S2). Because of the change of the nanostructures, the CA of a water droplet is increased to 132.4 degrees (inset of Fig. 2b), which is greatly enhanced compared with the vertical Si nanowires. These results mean that the conformation changes of Si nanowires etched under the DC electric field lead to a macroscopic change in CA.

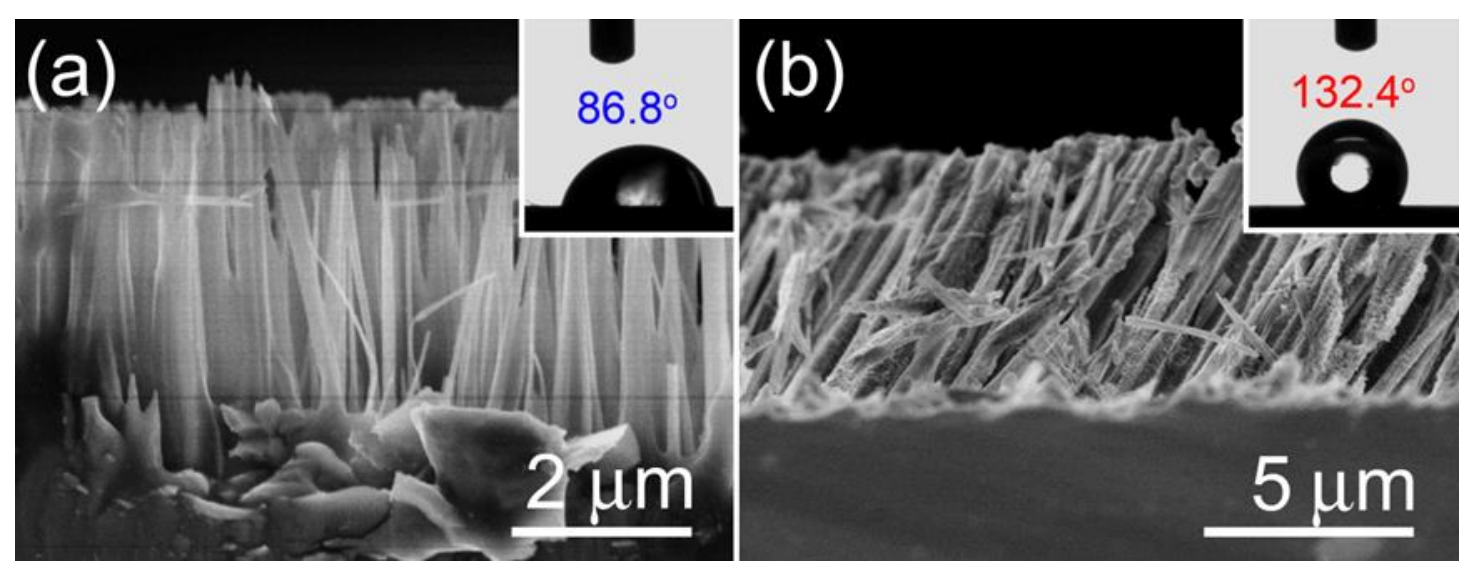

Fig. 2 Cross-sectional SEM images of Si nanowire samples. (a) the vertical $\mathrm{Si}$ nanowires, (b) Si nanowires etched under electric filed. Insets shows the CA of vertical and slant Si nanowires, respectively.

\subsection{Mechanism of the formation of Si nanoswires}

The formation mechanism can be interpreted with Si electrochemical reaction in HF solution [14], there are three half-reactions as follow:

$$
\begin{aligned}
& \mathrm{H}_{2} \mathrm{O}_{2}+2 \mathrm{H}^{+}+2 \mathrm{e}^{-} \rightarrow 2 \mathrm{H}_{2} \mathrm{O} \\
& \mathrm{Ag}^{+}+\mathrm{e}^{-} \rightarrow \mathrm{Ag} \\
& \mathrm{SiF}_{6}{ }^{2-}+4 \mathrm{e}^{-} \rightarrow \mathrm{Si}+6 \mathrm{~F}^{-}
\end{aligned}
$$

The etching of Si includes two main reactions as shown below: 


$$
\begin{aligned}
& \mathrm{Si}+4 \mathrm{Ag}^{+}+6 \mathrm{~F}^{-} \rightarrow 4 \mathrm{Ag}+\mathrm{SiF}_{6}^{2-} \\
& 2 \mathrm{Ag}+\mathrm{H}_{2} \mathrm{O}_{2}+2 \mathrm{H}^{+} \rightarrow 2 \mathrm{Ag}^{+}+2 \mathrm{H}_{2} \mathrm{O}
\end{aligned}
$$

The net etching reaction of reactions (1) to (5) is

$$
\mathrm{Si}+2 \mathrm{H}_{2} \mathrm{O}_{2}+6 \mathrm{~F}^{-}+4 \mathrm{H}^{+} \rightarrow \mathrm{SiF}_{6}^{2-}+4 \mathrm{H}_{2} \mathrm{O}
$$

Fig. 3 could be used to explain the etching process. In Fig. $3 a$ and $3 \mathrm{~b}, \mathrm{Ag}$ nanoparticles are coated on the surface of Si wafer by reaction 4. After removing of $\mathrm{Ag}^{+}$ion, the wafer is put into an etchant containing $\mathrm{HF}$ and $\mathrm{H}_{2} \mathrm{O}_{2}$. The $\mathrm{Ag}$ is oxidized immediately and partially dissolved in solution to form $\mathrm{Ag}^{+}$ions according to reactions 2 and 5. The $\mathrm{Ag}^{+}$ions quickly react with the $\mathrm{Si}$ at the interfaces between the wafer and the $\mathrm{Ag}$ nanoparticles by reaction 4, so the etching is localized in the regions covered by Ag and the Ag nanoparticles gradually decrease and go down. The process of net etching reaction 6 could be depicted in Fig. 3c, in which the $\mathrm{H}_{2} \mathrm{O}_{2}$ and $\mathrm{HF}$ diffuse into the pores and the $\mathrm{SiF}_{6}{ }^{2-}$ and $\mathrm{H}_{2} \mathrm{O}$ come out. With the continuous etching reaction, the Ag nanoparticles go deeper. Hence, the residual Si forms the Si nanowire arrays.

Fig. $3 \mathrm{~d}$ schematizes that the process of DC electric field influence the geometry on silicon. The slant structure can be viewed as the movement track of the metal catalyst, so controlling motion of metal catalyst is the key to control of the etching direction [15]. It was found that the motion direction of Ag catalyst particle could be influenced under the DC electric field. In the DC electric field, a movement of $\mathrm{Ag}^{+}$ could be occurred at horizontal direction, meantime, the formed Ag particles are subjected to a downward force at perpendicular direction, therefore the movement track of Ag is slant. With increasing the depth of etching, slant Si nanowire arrays were formed gradually. After removing Ag particles, plenty of pits were left on surface of Si nanowire which caused the rough surface of Si nanowires (Fig. S2). 


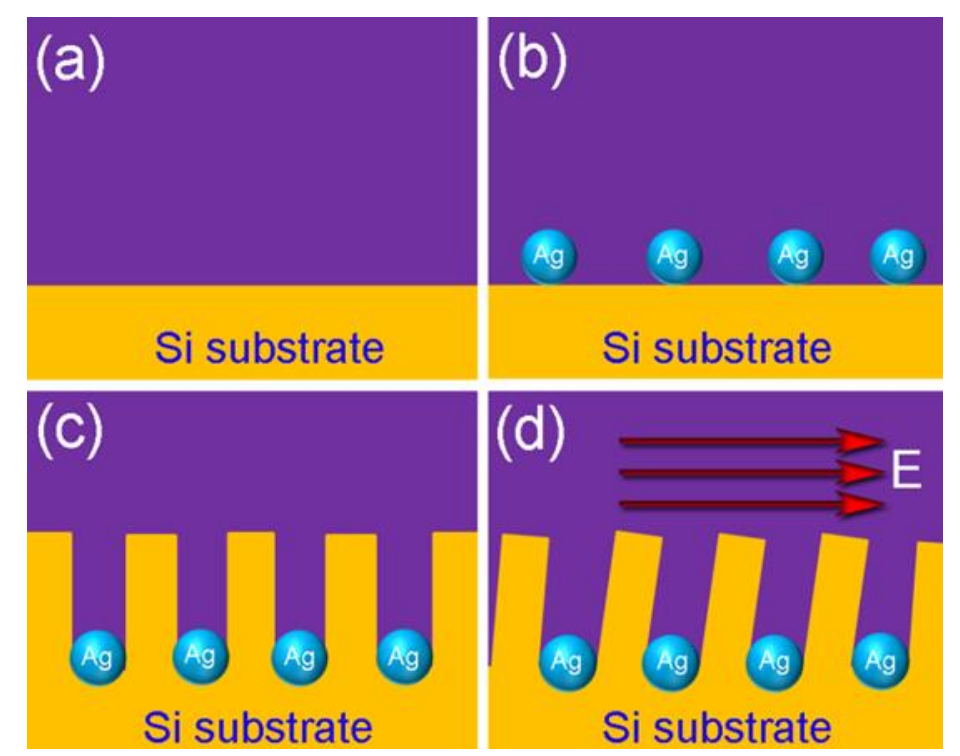

Fig. 3 Schematic of the fabrication process of Si nanowires (a) Si substrate immerse in etching solution, (b) Ag nanoparticles are generated in the etching solution, (c) vertical and (d) slant grown Si nanowires.

\section{Conclusion}

In summary, we have investigated the etching process of $\mathrm{Si}$ nanowires, and proposed a new method that introduced DC electric field to change the growth directions of Si nanowires. The results showed that the growth of Si nanowires under the electric field could become slant compare with no electric field applied. Meanwhile, the contact angle of slant Si nanowires was also increased compared with vertical Si nanowires $\left(132.4^{\circ}\right.$ vs. $\left.86.8^{\circ}\right)$, which was ascribed to the rough surface formed in the electric field. Importantly, it is possible that some other metal ions chemical etching could be also influenced by applied DC electric field or alternating current electric field, detailed studies need to be done in the near future for more exploring.

\section{Acknowledgements}

This work was financially supported by the National Natural Science Foundation of 
China (Grant No. 51602193). Shanghai University of Engineering Science Innovation Fund (16KY0512).

\section{References}

[1] F. Xia and L. Jiang, Bio-Inspired, Smart, Multiscale Interfacial Materials, Adv. Mater., 20 (2008), 2842-2858.

[2] L. Jiang, Y. Zhao and J. Zhai, A lotus-leaf-like superhydrophobic surface: a porous microsphere/nanofiber composite film prepared by electrohydrodynamics, Angew. Chem., Int. Ed., 43 (2004), 4338-4341.

[3] X. F. Gao and L. Jiang, Biophysics: water-repellent legs of water striders, Nature, 432 (2004), 36.

[4] Z. Guo, W. Liu and B.-L. Su, Superhydrophobic surfaces: From natural to biomimetic to functional, J. Colloid Interface Sci., 353 (2011), 335-355.

[5] P. Roach, N. J. Shirtcliffe and M. I. Newton, Progess in superhydrophobic surface development, Soft Matter, 4 (2008), 224-240.

[6] B. Bhushan and Y. C. Jung, Natural and biomimetic artificial surfaces for superhydrophobicity, self-cleaning, low adhesion, and drag reduction, Prog. Mater. Sci., 2011, 56, 1-108.

[7] K. S. Liu and L. Jiang, Metallic surfaces with special wettability, Nanoscale, 3 (2011), 825-838.

[8] X. X. Zhang, M. Chen, Influence of homogeneous electric field on the structure and growth of ice, Acta Phys. Chim. Sin., 30 (2014), 1208-1214.

[9] Y. Q. Zeng, B. Tao, J. K. Chen and Z. P. Yin, Study on external electric 
field-induced structural changes in the initial growth of pentacene on amorphous $\mathrm{SiO}_{2}$, J. Phys. D: Appl. Phys. 49 (2016) 195308.

[10]Ş. Ţălu, S. Solaymani, M. Bramowicz, S. Kulesza, A. Ghaderi, S. Shahpouri, S. M. Elahi, Effect of electric field direction and substrate roughness on three dimensional self-assembly growth of copper oxide nanowires, J Mater Sci: Mater Electron 27 (2016), 9272-9277

[11]Y. G. Lu, X. L. Liang, Y. Q. Gong, X. J. Zheng, Z. Z. Liu, Effect of external electric field on phase transitions of ferroelectric thin films, Acta Phys. Sin. 59 (2010), 8167-8171.

[12]Z. L. Zhao, B. Liu, R. Zhang, L. Liu, Investigation on Solidification of Metal in Electric Field, Mater. Rev. 9 (2001), 23-25.

[13]K. Peng, Y. Yan, S. Gao, J. Zhu, Dendrite-assisted growth of silicon nanowires in electroless metal deposition. Adv. Funct. Mater. 13 (2003), 127-132.

[14]M. L. Zhang, K. Q. Peng, X. Fan, J. S. Jie, R. Q. Zhang, S. T. Lee and N. B. Wong, Preparation of Large-Area Uniform Silicon Nanowires Arrays through Metal-Assisted Chemical Etching. J. Phys. Chem. C, 112 (2008), 4444-4450.

[15]X. Jiao, Y. Chao, L. Q. Wu, A. Q. Yao, Metal-assisted chemical etching of silicon 3D nanostructure using direct-alternating electric field. J. Mater. Sci.: Mater. El., 27 (2016), 1881-1887. 